\title{
Efecto de la flipped classroom en las estrategias de aprendizaje de los estudiantes universitarios
}

\author{
María Espada, José A. Navia, \\ Maite Gómez-López \& Patricia Rocu
}

\begin{abstract}
Resumen
En la actualidad están surgiendo nuevos métodos de enseñanza que permitan a los estudiantes disponer de mayor autonomía para la construcción de su propio aprendizaje. En este estudio se analiza la influencia de la flipped classroom en las estrategias de aprendizaje en el contexto universitario. Participaron un total de 96 estudiantes universitarios que fueron divididos en dos grupos, un grupo recibió una intervención con el método tradicional y otro grupo una intervención mediante clase invertida, se analizó las estrategias de aprendizaje del alumnado antes y después de la intervención. Los resultados de esta investigación muestran que la intervención tiene un efecto positivo en los participantes dado que sus puntuaciones aumentan con el tiempo, mostrando que con el método de flipped classroom los participantes mejoraron sus puntaciones con respecto al inicio. Además, los resultados mostraron un efecto principal para el tipo de enseñanza en la estrategia de manejo de recursos, donde la enseñanza tradicional obtuvo mejores puntuaciones. Respecto al sexo, existen diferencias estadísticamente significativas en estrategias de procesamiento de la información, obteniendo las mujeres mayores puntuaciones que los hombres.
\end{abstract}

Palabras clave:

clase invertida; método tradicional; estrategias de aprendizaje; alumnado universitarios; sexo 


\section{The flipped classroom effect on the learning strategies of university students}

Abstract: Currently emerging new methods of teaching, allowing students to have greater autonomy for the construction of their own learning. This study analyses the influence of a flipped classroom on learning strategies in the university context. A total of 96 university students participated in the research and were divided into two groups, one group received an intervention based on the traditional method and the other an intervention using the flipped classroom method; the students' learning strategies were subsequently analysed before and after the intervention. The results of this research show that the intervention has a positive effect on the participants given that their scores increase with the time, showing that while with the flipped classroom method participants improved their scores. Furthermore, the results showed a main effect for the type of teaching in the strategy of resource management, where the traditional teaching was awarded best scores. Regarding gender, there were statistically significant differences in the strategies to process information, where women obtained higher scores than men.

Keywords: flipped classroom; traditional method; learning strategies; university students; gender

\section{O efeito de aula invertida sobre as estratégias de aprendizagem dos estudantes universitários}

Resumo: Estão agora a surgir novos métodos de ensino que permitem aos estudantes uma maior autonomia na construção da sua própria aprendizagem. Este estudo analisa a influência da sala de aula invertida sobre as estratégias de aprendizagem no contexto universitário. Um total de 96 estudantes universitários participaram e foram divididos em dois grupos, um grupo recebeu uma intervenção com o método tradicional e o outro grupo recebeu uma intervenção através de uma aula invertida. As estratégias de aprendizagem dos estudantes foram analisadas antes e depois da intervenção. Os resultados desta investigação mostram que a intervenção tem um efeito positivo nos participantes à medida que as suas pontuações aumentam com o tempo, mostrando que com o método de aula invertida os participantes melhoraram as suas pontuações desde o início. Além disso, os resultados mostraram um efeito importante para o tipo de ensino na estratégia de gestão de recursos, onde o ensino tradicional teve uma melhor pontuação. No que respeita ao género, existem diferenças estatisticamente significativas nas estratégias de processamento de informação, com as mulheres a obterem pontuações mais elevadas do que os homens.

Palavras- chave: aula invertida; método tradicional; estratégias de aprendizagem; estudantes universitários; género

\section{Effet des stratégies d'apprentissage des élèves de collège flipped classroom}

Résumé: Actuellement émergent de nouvelles méthodes d'enseignement, permettant aux étudiants d'avoir une plus grande autonomie pour la construction de leur propre apprentissage. Cette étude traite de l'influence de la flipped classroom sur les stratégies d'apprentissage dans le contexte universitaire. Au total, 96 étudiants universitaires ont participé et ont été divisés en deux groups, un groupe a reçu une intervention de méthode traditionnelle et un autre groupe une intervention de classe inverse; les stratégies d'apprentissage des élèves ont été discutées avant et après l'intervention. Les résultats de cette recherche montrent que l'intervention a un effet positif sur les participants à mesure que leurs scores augmentent au fil du temps, montrant qu'avec la méthode de la salle de flipped classroom, les participants ont amélioré leurs conseils par rapport au début. En outre, les résultats ont montré un effet majeur sur le type d'enseignement dans la stratégie de gestion des ressources, où l'enseignement traditionnel a obtenu de meilleurs résultats. En ce qui concerne le genre, il existe des différences statistiquement significatives dans les stratégies de traitement de l'information, les femmes obtenant des scores plus élevés que les homes.

Mots clés: flipped classroom; méthode traditionnelle; stratégies d'apprentissage; étudiants universitaires; genre 


\section{Introducción}

El concepto de estrategias de aprendizaje ha ido evolucionando a lo largo de los años, pasando de una connotación únicamente cognitiva y metacognitiva, a integrar elementos afectivos-emocionales y de apoyo (Gargallo-López, Suárez-Rodríguez, \& Pérez-Pérez, 2009; Herrera-Torres \& Lorenzo-Quiles, 2009). Las estrategias de aprendizaje pueden entenderse como el conjunto organizado, consciente e intencional de lo que hace el aprendiz para lograr con eficacia un objetivo de aprendizaje en un contexto social dado (Gargallo-López et al., 2009).

Las diferentes leyes educativas (LOGSE, 1990, LOE, 2006, LOMCE, 2013), vienen planteando desde un enfoque constructivista la recomendación de dotar al discente de las competencias necesarias para que pueda construir y producir su propio conocimiento (Huéscar, Moreno-Murcia y Alias, 2017).

En este sentido, se pretende realizar un cambio en la metodología de enseñanza, dejando a un lado el método "tradicional" cuyo protagonismo reside en la figura del docente y en la mera transmisión de conocimientos a través de clases magistrales, la relación comunicativa que se establece en el aula es puramente transmisora, jerárquica y unidireccional (López, 2007).

En esta línea, la reforma actual de los estudios universitarios, incrementa, si cabe, la importancia de las estrategias de aprendizaje poniendo el énfasis en la autonomía del estudiante en la construcción de su propio conocimiento (López Aguado, 2011). Así, las Instituciones de Educación Superior han comenzado a articular sus herramientas pedagógicas a la enseñanza de las diferentes áreas del saber (Vélez, Palacio, Hernández, Ortiz y Gaviria, 2019).

Este cambio educativo también hace especial hincapié en la utilización de la tecnología para el aprendizaje. En esta línea, las herramientas tecnológicas que los estudiantes tienen a su disposición les ofrecen opciones para lograr que el aula tradicional se convierta en un nuevo espacio, en donde tienen a su disposición actividades innovadoras de carácter colaborativo y con aspectos creativos que les permiten afianzar lo que aprenden al mismo tiempo que se divierten (Hernández Requena, 2008). Diferentes investigaciones tales como la de Mothibi (2015) concluyen que la utilización de estas herramientas tecnológicas tiene un impacto positivo en el aprendizaje de los estudiantes. Todo ello ha llevado al ámbito educativo a integrar estas herramientas tecnológicas y desarrollar nuevos métodos de enseñanza.

Entre esos nuevos métodos, se encuentra la flipped classroom que sin duda revoluciona el escenario educativo, ya que los estudiantes pueden ver los contenidos del tema a través del aula virtual y posteriormente en la clase realizar actividades y ejercicios prácticos que permitan consolidar esos contenidos. El aula se convierte en un lugar de experiencias prácticas en la que el alumnado es el protagonista de su proceso 
de aprendizaje. Sin embargo, a día de hoy no existe suficientes investigaciones científicas que permitan avalar la idoneidad de la clase invertida como método en todas las áreas y niveles educativos (Abeysekera \& Dawson, 2015; Kashada, Li, \& Su, 2017).

En el contexto de los estudios universitarios existen diversas investigaciones que relacionan las estrategias de aprendizaje con el rendimiento académico (Camarero Suárez, Buey, \& Herrero Díez, 2000; Gargallo López, Suárez Rodríguez, \& Ferreras Remesal, 2007; Martín, García, Torbay, \& Rodríguez, 2008). Sin embargo, son aún escasas las investigaciones que analizan la relación entre los métodos de enseñanza utilizados y las estrategias de aprendizaje de los estudiantes (Gargallo-López, Pérez-Pérez, Verde-Peleato, \& García-Félix, 2017).

Por ello, el objetivo del presente trabajo es analizar si la metodología flipped classroom influye en las estrategias de aprendizaje de los estudiantes universitarios.

\section{Método}

\section{Participantes}

En este estudio se trabajó, durante el primer semestre del curso 2017-2018, en una asignatura de carácter obligatorio del tercer curso del Grado en Ciencias de la Actividad Física y del Deporte, concretamente en la asignatura de Metodología aplicada a las en Ciencias de la Actividad Física y del Deporte durante una unidad didáctica. La muestra total fue de 96 estudiantes, de los cuales el $78.1 \%$ eran hombres y el $21.9 \%$ mujeres, con una edad media de 21.7 años $(D S=3.21)$.

\section{Material y procedimiento}

Para poder medir las estrategias de aprendizaje en estudiantes universitarios se utilizó el cuestionario elaborado por Gargallo-López et al. (2009). Este instrumento consta de dos escalas, seis subescalas, veinticinco estrategias y 88 ítems, la fiabilidad del instrumento es de alfa de Cronbach $=.89$.

Se realizaron mediciones en cada uno de los grupos utilizando el instrumento mencionado anteriormente. Para la intervención se distribuyó al alumnado en dos grupos, uno de ellos recibió los contenidos a través del método de clase tradicional, es decir, con clases magistrales impartidas por el docente y resolución de dudas en la clase. El otro grupo, recibió los contenidos utilizando el método de flipped classroom a través de vídeos que estaban subidos al espacio virtual de la asignatura y el alumnado tenía que ver fuera del horario de clase, posteriormente durante el horario de clase recibieron sesiones prácticas en las que se trataba los contenidos del aula virtual y se podían resolver las posibles dudas sobre los contenidos abordados en los vídeos. 


\section{Diseño y análisis de datos}

Se trata de un diseño experimental, en el que un grupo recibió una intervención pedagógica tradicional y otro grupo una intervención mediante clase invertida. A ambos grupos se les registraron sus percepciones antes y después de la intervención pedagógica. El diseño por tanto responde a una estructura de 2 grupos (tradicional vs invertida) $\times 2$ tiempos (antes y después). Las variables dependientes fueron los valores resultantes que componen las diferentes estrategias, subescalas y escalas del cuestionario (ver Gargallo et al., 2009). Estas puntuaciones se analizaron mediante un Análisis de Varianza de Medidas Repetidas (ANOVA MR), introduciendo el tiempo como factor intra-grupo y el tipo de enseñanza como factor inter-grupo. Se controló la igualdad de varianzas mediante la prueba de Levene. Las puntuaciones originales de aquellas variables que violaban este supuesto se transformaron exponencialmente (ver pie de Tabla 1) y se analizaron de igual forma con las nuevas puntuaciones. El tamaño del efecto se expresó mediante eta cuadrado parcial (np2), con valores de 0.01, 0.06, y 0.14 para tamaños pequeños, medianos y grandes, respectivamente (Cohen, 1988). Se utilizó el programa SPSS V.24 y jamovi V. 0.9.5.16 para realizar los análisis estadísticos y generar gráficos

\section{Resultados}

Como se puede observar en la Tabla 1, las puntuaciones se encontraban en un rango entre 2.0 y 4.3, con desviaciones típicas que fluctuaban entre 0,2 a 1,2 puntos. En términos generales, se observa que la intervención tiene un efecto positivo en los participantes dado que sus puntuaciones aumentan con el tiempo. De hecho, en 14 de las 25 estrategias identificadas, en todas las subescalas, en las dos escalas así como en el total, el factor tiempo tuvo un efecto principal sobre las puntuaciones (Tabla 2).

El ANOVA MR también reveló interacciones entre el tiempo y el tipo de enseñanza para las estrategias de Personalización y creatividad, y de Selección de información, así como para la subescala de Búsqueda de información (Tabla 2). Esta interacción indicó que mientras con la enseñanza invertida los participantes mejoraron sus puntaciones con respecto al inicio, con la enseñanza tradicional no (Tabla 1). Por último, el análisis mostró un efecto principal para el tipo de enseñanza en la estrategia de Manejo de recursos (Tabla 2), donde en términos generales la enseñanza tradicional obtuvo mejores puntuaciones (Tabla 1). No se encontraron más efectos principales ni interacciones en los análisis. 
Tabla 1. Media (M) y Desviación Típica (DT) de las diferentes estrategias, subescalas, escalas y el total del cuestionario CEVEAPEU por tipo de enseñanza y por tiempo

\begin{tabular}{|c|c|c|c|c|c|c|c|c|}
\hline & \multicolumn{4}{|c|}{ Tradicional $\mathrm{n}=39$} & \multicolumn{4}{|c|}{ Invertida $n=57$} \\
\hline & \multicolumn{2}{|c|}{ Antes } & \multicolumn{2}{|c|}{ Después } & \multicolumn{2}{|c|}{ Antes } & \multicolumn{2}{|c|}{ Después } \\
\hline & $M$ & $D T$ & $M$ & $D T$ & $M$ & $D T$ & $M$ & $D T$ \\
\hline \multicolumn{9}{|l|}{ Estrategias } \\
\hline Planificación & 3,0 & 0,5 & 3,2 & 0,7 & 3,1 & 0,6 & 3,2 & 0,6 \\
\hline Habilidades de interacción social & 3,8 & 0,5 & 4,0 & 0,5 & 3,7 & 0,6 & 3,9 & 0,5 \\
\hline Autoeficacia y Expectativas & 4,3 & 0,6 & 4,2 & 0,6 & 4,2 & 0,6 & 4,2 & 0,6 \\
\hline Ansiedad & 2,9 & 0,6 & 3,1 & 0,6 & 2,9 & 0,6 & 3,0 & 0,7 \\
\hline Esta do Fisico y Animico & 3,7 & 0,7 & 3,8 & 0,6 & 3,6 & 0,7 & 3,7 & 0,6 \\
\hline Valor de la tarea & 4,0 & 0,6 & 4,1 & 0,5 & 3,9 & 0,6 & 3,9 & 0,7 \\
\hline Control del contexto & 3,8 & 0,6 & 3,8 & 0,5 & 3,8 & 0,7 & 4,0 & 0,8 \\
\hline Control y Autorregulación & 3,8 & 0,5 & 3,9 & 0,5 & 3,7 & 0,6 & 3,8 & 0,6 \\
\hline Atribuciones Internas & 4,0 & 0,5 & 4,2 & 0,4 & 4,2 & 0,5 & 4,2 & 0,5 \\
\hline Atribuciones Externas & 2,4 & 0,8 & 2,8 & 1,0 & 2,6 & 0,9 & 2,8 & 0,8 \\
\hline Conocimiento y criterios & 3,6 & 0,8 & 3,6 & 0,7 & 3,4 & 0,8 & 3,5 & 1,0 \\
\hline $\begin{array}{l}\text { Concepción de la inteligencia como } \\
\text { modificable }\end{array}$ & 3,1 & 0,6 & 3,3 & 0,6 & 3,2 & 0,6 & 3,3 & 0,6 \\
\hline Motivación Extrinseca & 2,0 & 1,1 & 2,3 & 1,2 & 2,1 & 0,9 & 2,4 & 1,0 \\
\hline Autoevaluación & 4,1 & 0,4 & 4,0 & 0,4 & 4,0 & 0,6 & 3,9 & 0,6 \\
\hline Motivación Intrinseca & 4,1 & 0,6 & 4,1 & 0,5 & 4,0 & 0,6 & 4,0 & 0,6 \\
\hline Organización de la información & 3,7 & 0,8 & 3,8 & 0,8 & 3,3 & 0,9 & 3,5 & 0,9 \\
\hline Personalización y Creatividad & 3,7 & 0,7 & 3,7 & 0,6 & 3,4 & 0,8 & 3,6 & 0,7 \\
\hline Adquisición Informaciones & 2,8 & 0,8 & 3,1 & 0,8 & 2,8 & 0,8 & 3,1 & 1,0 \\
\hline Elaboración de información & 4,1 & 0,5 & 4,0 & 0,6 & 3,9 & 0,7 & 3,9 & 0,6 \\
\hline Almacenamiento y memorización & 3,6 & 0,9 & 3,9 & 0,8 & 3,6 & 1,1 & 3,7 & 0,9 \\
\hline Fuentes y búsqueda de info. & 3,3 & 0,6 & 3,3 & 0,6 & 3,3 & 0,8 & 3,5 & 0,8 \\
\hline Selección de Información & 3,6 & 0,4 & 3,6 & 0,5 & 3,4 & 0,5 & 3,7 & 0,6 \\
\hline Transferencia y uso de la información & 4,0 & 0,6 & 4,2 & 0,5 & 3,8 & 0,8 & 4,0 & 0,7 \\
\hline Almacenamiento y repetición & 2,6 & 1,0 & 2,9 & 1,0 & 2,4 & 1,0 & 2,6 & 1,0 \\
\hline Manejo de recursos & 3,5 & 0,7 & 3,8 & 0,7 & 3,2 & 0,8 & 3,5 & 0,9 \\
\hline \multicolumn{9}{|l|}{ Subescalas } \\
\hline Estrategias motivacionales & 3,4 & 0,3 & 3,6 & 0,4 & 3,5 & 0,3 & 3,5 & 0,3 \\
\hline Componentes afectivos & 3,3 & 0,5 & 3,5 & 0,4 & 3,3 & 0,4 & 3,3 & 0,5 \\
\hline Estrategias metacognitivas & 3,6 & 0,3 & 3,7 & 0,4 & 3,6 & 0,4 & 3,6 & 0,5 \\
\hline Contexto e interacción social & 3,8 & 0,4 & 3,9 & 0,3 & 3,8 & 0,5 & 3,9 & 0,5 \\
\hline Búsqueda de información & 3,5 & 0,2 & 3,7 & 0,3 & 3,5 & 0,3 & 3,6 & 0,3 \\
\hline Procesamiento y uso de la inform. & 3,5 & 0,4 & 3,5 & 0,5 & 3,4 & 0,6 & 3,6 & 0,6 \\
\hline \multicolumn{9}{|l|}{ Escalas } \\
\hline A fectivas, apoyo y control & 3,5 & 0,4 & 3,7 & 0,4 & 3,3 & 0,5 & 3,5 & 0,5 \\
\hline Procesamiento de la información & 3,5 & 0,3 & 3,6 & 0,4 & 3,3 & 0,5 & 3,5 & 0,5 \\
\hline Total & 3,5 & 0,2 & 3,6 & 0,3 & 3,4 & 0,4 & 3,6 & 0,4 \\
\hline
\end{tabular}


Tabla 2. Diferencias significativas en función del tiempo (antes vs después) y tipo de enseñanza (tradicional vs invertida), e interacciones significativas entre estos dos factores.

\begin{tabular}{|c|c|c|c|c|c|c|c|c|c|}
\hline & \multicolumn{3}{|c|}{ Tiempo } & \multicolumn{3}{|c|}{ Tipo de enserianza } & \multicolumn{3}{|c|}{ Interacción } \\
\hline & $F$ & $p$ & $\eta_{p}^{2}$ & $F$ & $p$ & $\eta_{p}^{2}$ & $F$ & $p$ & $\eta_{p}^{2}$ \\
\hline \multicolumn{10}{|l|}{ Estrategias } \\
\hline Planificación & 5,26 & 0,024 & 0,05 & & & & & & \\
\hline Habilidades interacción & 12,15 & $<001$ & 0,11 & & & & & & \\
\hline Ansiedad & 5,72 & 0,019 & 0,06 & & & & & & \\
\hline A tribuciones extemas & 11,51 & 0,001 & 0,02 & & & & & & \\
\hline Concep intelig. modific. & 6,58 & 0,012 & 0,07 & & & & & & \\
\hline Motivación extrinseca & 4,24 & 0,006 & 0,08 & & & & & & \\
\hline Organización de la info. & 5,14 & 0,026 & 0,05 & & & & & & \\
\hline Personalización y creativ. & 4,14 & 0,044 & 0,04 & & & & 4,67 & 0,033 & 0,05 \\
\hline Adquisición de info & 12,37 & $<001$ & 0,12 & & & & & & \\
\hline Fuentes y búsqueda biblio ${ }^{\mathrm{a}}$ & 4,26 & 0,042 & 0,04 & & & & & & \\
\hline Selección de información & 6,51 & 0,012 & 0,06 & & & & 4,83 & 0,030 & 0,05 \\
\hline Transferencia y uso info ${ }^{\mathrm{a}}$ & 4,26 & 0,042 & 0,04 & & & & & & \\
\hline Almacenam. y repetición & 6,02 & 0,016 & 0,06 & & & & & & \\
\hline Manejo de recursos & 17,00 & $<001$ & 0,15 & 4,33 & 0,040 & 0,04 & & & \\
\hline \multicolumn{10}{|l|}{ Subescalas } \\
\hline Estrategias motivacionales & 15,89 & $<, 001$ & 0,14 & & & & & & \\
\hline Componentes afectivos & 7,00 & 0,010 & 0,07 & & & & & & \\
\hline Estrategias metacognitivas & 4,88 & 0,030 & 0,05 & & & & & & \\
\hline Estr contexto-interacción & 10,38 & 0,002 & 0,10 & & & & & & \\
\hline Búsqueda de información ${ }^{\mathrm{a}}$ & 10,13 & 0,002 & 0,10 & & & & 4,99 & 0,028 & 0,05 \\
\hline Procesamiento de la info. & 28,49 & $<, 001$ & 0,23 & & & & & & \\
\hline \multicolumn{10}{|l|}{ Escalas } \\
\hline Afectivas, apoyo y control & 24,18 & $<001$ & 0,20 & & & & & & \\
\hline Procesamiento info. & 25,95 & $<, 001$ & 0,22 & & & & & & \\
\hline Total $^{b}$ & 36,68 & $<001$ & 0,28 & & & & & & \\
\hline
\end{tabular}

$a=$ transformación al cuadrado, b=transformación a la quinta potencia 
Finalmente, con el objetivo de ver si los chicos y las chicas se comportaban de la misma forma o por el contrario había algún tipo de diferencia entre ellos, se analizaron las subescalas, escalas y el total del cuestionario añadiendo como factor intergrupal la variable sexo: 2 tiempos $\times 2$ tipos de enseñanza $\times 2$ sexos. Los resultados, aparte de confirmar los efectos principales del factor tiempo (ver Tabla 1), mostraron un efecto principal del factor sexo en la subescala de Estrategias de procesamiento y uso de la información, $F(1,92)=3,96, p=0,049$, np2 = 0,04. Es decir, las chicas puntuaron más alto que los chicos en I3as estrategias de procesamiento de la información (Figura 1). Además, el ANOVA MR reveló dos interacciones significativas entre el sexo y el tipo de enseñanza para la subescala de componentes afectivos, $F(1,92)=7,86, p=0,006$, $\eta p 2=0,08$ y para la escala de estrategias afectivas, de apoyo y control, $F(1,92)=4,95$, $p=0,028, \eta p 2=0,05$. En ambos casos los valores de las chicas son más altos en la clase invertida y más bajos en la clase tradicional con respecto a los chicos, y viceversa (Figura 2). No se encontraron en los resultados otras diferencias significativas por sexo o interacciones entre sexo y tiempo o entre sexo y tipo de enseñanza.
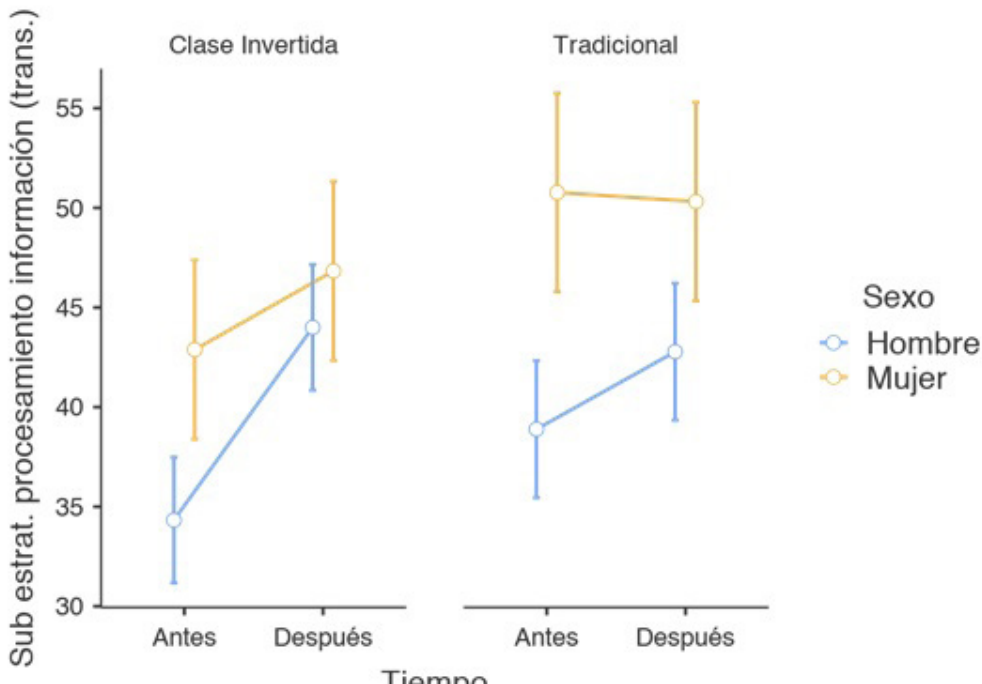

Figura 1. Puntuaciones transformadas de la subescala Estrategias de procesamiento de la información en función del tiempo, del tipo de enseñanza y del sexo. Las barras de error indican el error estándar de la media. Las puntuaciones originales se transformaron mediante la tercera potencia de los valores originales. 

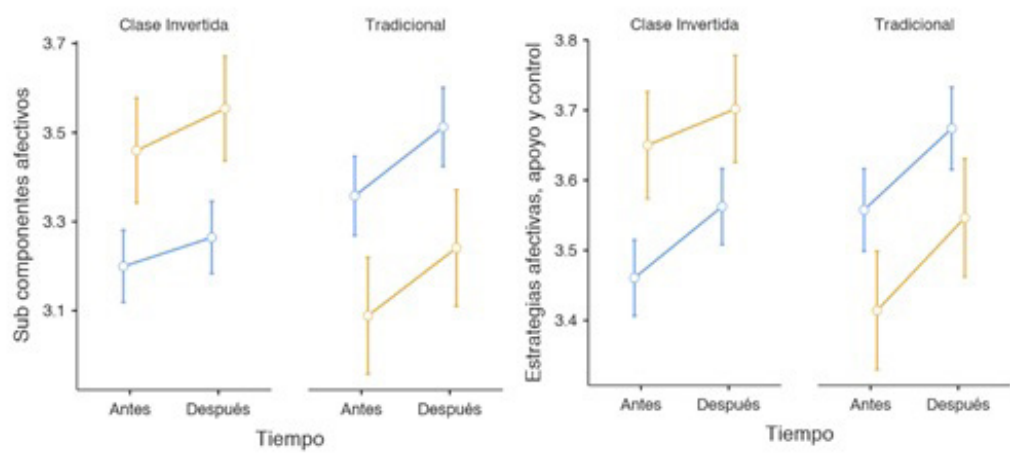

Sexo

Hombre

Mujer

Figura 2. Puntuaciones de la subescala Componentes Afectivos y de la Escala Estrategias afectivas, de apoyo y control en función del tiempo, del tipo de enseñanza y del sexo. Las barras de error indican el error estándar de la media.

\section{Discusión}

En el presente trabajo se pretende analizar si la metodología flipped classroom influye en las estrategias de aprendizaje de los estudiantes universitarios. Para poder evaluar las estrategias de aprendizaje, se han analizado dos escalas: estrategias afectivas, apoyo y control y estrategias de procesamiento de la información (Gargallo-López et al., 2009).

En cuanto a la escala de estrategias afectivas, apoyo y control, a nivel general observamos que no existen apenas diferencias entre el alumnado que ha recibido la intervención a través del método tradicional y los que han seguido el método flipped classroom, ya que los primeros obtuvieron una puntuación antes de la intervención de $M=3.5$; $D T=04$, y después de $M=3.7$; $D T=0.24$; y los que han seguido el método flipped classroom alcanzaron una $M=3.3$; $D T=0.5$ antes de la intervención y de $M=3.5$; DT=0.5 después. Si profundizamos en el análisis, observamos que existen diferencias de medias en las diferentes subescalas; estrategias motivacionales, componentes afectivos, estrategias metacognitivas y contexto e interacción social, ya que los estudiantes en los que se ha aplicado el método tradicional aumenta ligeramente sus puntuaciones medias en todas las subescalas después de la intervención, mientras que el alumnado del método flipped classroom únicamente aumentan la media de la escala contexto e interacción social tras realizar la intervención. Estos datos no coinciden con el estudio elaborado por Turan \& Gökta (2018) en el que el alumnado universitario que siguió el método de flipped classroom mostró una motivación superior al alumnado que siguió el método tradicional. 
Respecto a la escala de estrategias de procesamiento de la información, los resultados muestran que a nivel general no hay apenas diferencias entre el alumnado que recibió la intervención a través del método tradicional, alcanzando una $M=3.5 ; D T=0.3$ antes de la intervención y M=3.6; DT=0.4 después; y los del método flipped classroom que tenían una $\mathrm{M}=3.3$; $\mathrm{DT}=0.4$ antes de la intervención y $\mathrm{M}=3.5$; $\mathrm{D} 3=0.5$ después de ésta. Coincidiendo con la investigación de Gargallo-López et al. (2015) en la cual se analizó el efecto de la utilización de una metodología innovadora en las estrategias de aprendizaje de los estudiantes universitarios, y obtuvieron que no había apenas diferencias en la escala de estrategias de procesamiento de la información antes $(M=3.50$; $\mathrm{DT}=0.31)$ y después $(\mathrm{M}=3.66$; $\mathrm{DT}=0.44)$ de la intervención.

Al indagar en los resultados del presente estudio, observamos que existen diferencias estadísticamente significativas en las interacciones entre el tiempo y el tipo de enseñanza en la subescala búsqueda de información ( $p=.02)$ y en las estrategias de personalización y creatividad $(p=.03)$ y selección de información $(p=.03)$ revelando que mientras con la enseñanza invertida los participantes mejoraron sus puntaciones con respecto al inicio, con la enseñanza tradicional no. Sin embargo, el análisis mostró un efecto principal para el tipo de enseñanza en la estrategia de manejo de recursos $(p=0.04)$, donde en términos generales la enseñanza tradicional obtuvo mejores puntuaciones que el método de flipped classroom. Estos datos podrían deberse a que el alumnado no está familiarizado con el método flipped classroom y esto les lleva a invertir más tiempo para poder manejar y utilizar la información sobre los contenidos tratados de manera eficiente (Kim, 2018; Zainuddin \& Attaran, 2016).

Respecto a las posibles interacciones entre sexo y estrategias de aprendizaje, los resultados de la presente investigación muestran que, en la escala de estrategias de procesamiento de la información, si hay diferencias estadísticamente significativas en función del sexo $(p=.04)$, teniendo las mujeres mayor puntuación que los hombres. Esto quiere decir que las mujeres disponen de mayor habilidad para la adquisición, codificación, elaboración y organización de la información, personalización y creatividad, repetición y almacenamiento, recuperación de la información y estrategias de comunicación, uso de la información adquirida y transferencia (Gargallo López, 2012).

Los resultados revelaron dos interacciones significativas entre el sexo y el tipo de enseñanza para la subescala de componentes afectivos $(p=.00)$ y para la escala de estrategias afectivas, de apoyo y control $(p=.02)$. Siendo en ambos casos los valores de las chicas más altos en la clase invertida y más bajos en la clase tradicional con respecto a los chicos. Estos datos coinciden con diferentes estudios, en los que se concluye que las mujeres utilizan en mayor medida que los hombres estrategias afectivas y de apoyo social (Feldman et al., 2008; Hong-Nam \& Leavell, 2006; Yılmaz, 2010).

Finalmente, podemos concluir que la intervención tiene un efecto positivo en los participantes dado que sus puntuaciones aumentan con el tiempo, no obstante los 
resultados del presente estudio muestran que no existen muchas diferencias en las estrategias de aprendizaje de los estudiantes universitarios en función del método de enseñanza, flipped classroom o clase tradicional, el hecho de apelar al uso de estrategias no depende exclusivamente del estudiante; es decir, habría márgenes de acción que el docente podría capitalizar para favorecer el uso de estrategias por parte del alumnado (Rinaudo, Chiecher, \& Donolo, 2003).

En este sentido, la utilización por parte del profesorado de planteamientos centrados en el aprendizaje y la utilización de metodologías de enseñanza y evaluación adecuadas, permiten que el alumnado utilice más estrategias y de mayor calidad, al contrario de lo que ocurre cuando los profesores se suscriben a planteamientos centrados en la enseñanza a través de metodología tradicional y un examen final como método de evaluación (Gargallo López, Almerich, Suárez-Rodríguez, \& García-Félix, 2012).

Si bien, los datos de la presente investigación nos permiten reflexionar acerca de la importancia de la figura del docente para el desarrollo de estos nuevos métodos, clasificados como métodos activos. El método de flipped classroom podría no circunscribirse dentro de dichos métodos, ya que puede considerarse como una metodología expositiva, centrado en la figura del profesor, el cual imparte los contenidos de forma magistral, pero a través de una pantalla.

Es por ello, que consideramos necesario realizar más investigaciones que permitan analizar la puesta en práctica de diferentes métodos de enseñanza para poder valorar qué métodos permiten el uso de más estrategias y de mayor calidad por parte del alumnado universitarios.

\section{Agradecimientos:}

Este manuscrito forma parte de un proyecto de innovación educativa financiado por la Universidad Politécnica de Madrid, cuya referencia es: IE1718.1102.

\section{Referencias}

Abeysekera, L., \& Dawson, P. (2015). Motivation and cognitive load in the flipped classroom: definition, rationale and a call for research. Higher Education Research \& Development, 34(1), 1-14. doi: 10.1080/07294360.2014.934336

Camarero Suárez, F., Buey, F. M. d., \& Herrero Díez, J. (2000). Estilos y estrategias de aprendizaje en estudiantes universitarios. Psicothema, 12(4), 615-622.

Cohen, J. (1988). Statistical Power Analysis for the Behavioral Sciences. Second Edition. United States of America: Lawrence Erlbaum Associates.

Feldman, L., Goncalves, L., Chacón-Puignau, G., Zaragoza, J., Bagés, N., \& de Pablo, J. (2008). Relaciones entre estrés académico, apoyo social, salud mental y rendimiento académico en estudiantes universitarios venezolanos. Universitas Psychologica, 7(3), 739-751. 
Gargallo-López, B., Morera, I., \& García, E. (2015). Metodología innovadora en la universidad: sus efectos sobre los procesos de aprendizaje de los estudiantes universitarios. Anales de Psicología, 31, 901-915.

Gargallo-López, B., Pérez-Pérez, C., Verde-Peleato, I., \& García-Félix, E. (2017). Estilos de aprendizaje en estudiantes universitarios y enseñanza centrada en el aprendizaje. RELIEVE. Revista Electronica de Investigacion y Evaluacion, 23(2) art.2.http://doi.org/10.7203/relieve.23.2.9078

Gargallo-López, B., Suárez-Rodríguez, J. M., \& Pérez-Pérez, C. (2009). El cuestionario CEVEAPEU. Un instrumento para la evaluación de las estrategias de aprendizaje de los estudiantes universitarios. RELIEVE. Revista Electrónica de Investigación y Evaluación Educativa, 15(2), 1-31.

Gargallo López, B. (2012). Un aprendiz estratégico para una nueva sociedad. Teoría de la Educación. Educación y Cultura en la Sociedad de la Información, 13(2), 246-272.

Gargallo-López, B., Almerich, G., Suárez-Rodríguez, J. M., \& García-Félix, E. (2012). Estrategias de aprendizaje en estudiantes universitarios excelentes y medios. Su evolución a lo largo del primer año de carrera. RELIEVE. Revista Electrónica de Investigación y Evaluación Educativa, 18(2).

Gargallo López, B., Suárez Rodríguez, J., \& Ferreras Remesal, A. (2007). Estrategias de aprendizaje y rendimiento académico en estudiantes universitarios. Revista de Investigación Educativa, 25(2), 421-441.

Hernández Requena, S. (2008). El modelo constructivista con las nuevas tecnologías: aplicado en el proceso de aprendizaje. RUSC. Universities and Knowledge Society Journal, 5(2), 26-35.

Herrera-Torres, L., \& Lorenzo-Quiles, O. (2009). Estrategias de aprendizaje en estudiantes universitarios. Un aporte a la construcción del Espacio Europeo de Educación Superior. Educación y Educadores, 12(3), 75-98.

Hong-Nam, K., \& Leavell, A. G. (2006). Language learning strategy use of ESL students in an intensive English learning context. System, 34(3), 399-415. doi: https://doi.org/10.1016/j.system.2006.02.002

Huéscar, E., Moreno-Murcia, J.A. \& Alias, A. (2017). Diseño y validación de una escala para medir el estilo controlador del estudiante de Educación Superior. Psychology, Society, \& Education, 9(2), 239-249. doi: 10.25115/psye.v9i2.696

Kashada, A., Li, H., \& Su, C. (2017). Adoption of Flipped Classrooms in K-12 Education in Developing Countries: Challenges and Obstacles. [Emerging technologies; educational technology; electronic learning; flipped classrooms; K-12 education]. 12(10), 11. doi: 10.3991/ijet.v12i10.7308

Kim, J.Y. (2018). A study of students' perspectives on a flipped learning model and associations among personality, learning styles and satisfaction. Innovations in Education and Teaching International, 55(3), 314-324. doi: 10.1080/14703297.2017.1286998

López, F. (2007). Metodología participativa en la Enseñanza Universitaria. Narcea S.S. De Educiones: Madrid.

López Aguado, M. (2011). Estrategías de aprendizaje en estudiantes universitarios. Diferencias por género, curso y tipo de titulación. Teoría de la Educación. Educación y Cultura en la Sociedad de la Información, 12(2), 203-234. 
Martín, E., García, L. A., Torbay, Á., \& Rodríguez, T. (2008). Estrategias de aprendizaje y rendimiento académico en estudiantes universitarios. International Journal of Psychology and Psychological Therapy, 8(3), 401-412.

Martín, S. N. (2011). Principios, métodos y técnicas esenciales para la investigación educativa: Dykinson.

Mothibi, G. (2015). A Meta-Analysis of the Relationship between E-Learning and Students' Academic Achievement in Higher Education. Journal of Education and Practice, 6(9), 6-9.

Rinaudo, M. C., Chiecher, A., \& Donolo, D. (2003). Motivación y uso de estrategias en estudiantes universitarios. Su evaluación a partir del Motivated Strategies Learning Questionnaire. Anales de Psicología, 19(1), 107-119.

Turan, Z., \& Göktaş, Y. (2018). Innovative Redesign of Teacher Education ICT Courses: How Flipped Classrooms Impact Motivation? Journal of Education and Future, 13, 133-144.

Vélez, O., Palacio, S. M., Hernández, Y. L., Ortiz, P. A. y Gaviria, L. F. (2019). Aprendizaje basado en juegos formativos: caso Universidad en Colombia. Revista Electrónica de Investigación Educativa, 21, (12), 1-10. doi:10.24320/redie.2019.21.e12.2024

YIImaz, C. (2010). The relationship between language learning strategies, gender, proficiency and selfefficacy beliefs: a study of ELT learners in Turkey. Procedia - Social and Behavioral Sciences, 2(2), 682-687. doi: https://doi.org/10.1016/j.sbspro.2010.03.084

Zainuddin, Z., \& Attaran, M. (2016). Malaysian students' perceptions of flipped classroom: a case study. Innovations in Education and Teaching International, 53(6), 660-670. doi: 10.1080/14703297.2015.1102079

María Espada

Facultad de Ciencias de la Actividad Física y del Deporte (INEF. Universidad

Politécnica de Madrid.

Calle Martín Fierro, 7, 28040, Madrid, España.

Email: maria.espada@upm.es

ORCID: 0000-0002-3815-1037

José A. Navia

Facultad de Ciencias de la Actividad Física y del Deporte (INEF. Universidad

Politécnica de Madrid.

Calle Martín Fierro, 7, 28040, Madrid, España

Email:joseantonio.navia@upm.es

ORCID: 0000-0003-1218-5033

Maite Gómez-López

Facultad de Ciencias de la Actividad Física y del Deporte (INEF. Universidad

Politécnica de Madrid.

Calle Martín Fierro, 7, 28040, Madrid, España

Email: maitegomez.lopez@upm.es

ORCID: 0000-0002-8223-2053 
Patricia Rocu

Facultad de Ciencias de la Actividad Física y del Deporte (INEF. Universidad

Politécnica de Madrid.

Calle Martín Fierro, 7, 28040, Madrid, España

Email:patricia.rocu@upm.es

ORCID: 0000-0002-0016-4828

Correspondencia:

María Espada

Universidad Politécnica de Madrid, Facultad de Ciencias de la Actividad

Física y del Deporte. Calle Martín Fierro, 7, 28040, Madrid, España.

Data de submissão: Setembro 2019

Data de avaliação: Dezembro 2019

Data de publicação: Novembro 2020 